\title{
VINCI, the VLTI Commissioning Instrument: status after one year of operations at Paranal
}

\author{
Pierre Kervella $^{\mathrm{a}^{*}}$, Philippe Gitton ${ }^{\mathrm{a}}$, Damien Segransan ${ }^{\mathrm{c}}$, Emmanuel Di Folco $^{\mathrm{b}}$, Pierre Kern ${ }^{\mathrm{d}}$, \\ Mario Kiekebush ${ }^{\mathrm{a}}$, Thanh Phan Duc ${ }^{\mathrm{b}}$, Antonio Longinotti ${ }^{\mathrm{b}}$, Vincent Coudé du Foresto $^{\mathrm{e}}$, \\ Pascal Ballester ${ }^{\mathrm{b}}$, Cyrus Sabet ${ }^{\mathrm{b}}$, William D. Cotton ${ }^{\mathrm{f}}$, Markus Schöller $^{\mathrm{a}}$, Rainer Wilhelm ${ }^{\mathrm{b}}$ \\ ${ }^{a}$ ESO Chile, ${ }^{b}$ ESO Germany, ${ }^{\mathrm{c}}$ Observatoire de Genève, Switzerland, ${ }^{\mathrm{d}}$ LAOG, Observatoire de \\ Grenoble, France, ${ }^{e}$ Observatoire de Paris, France, ${ }^{f}$ National Radio Astronomy Observatory, USA
}

\begin{abstract}
Installed at the heart of the Very Large Telescope Interferometer (VLTI), VINCI combines coherently the infrared light coming from two telescopes. The first fringes were obtained in March 2001 with the VLTI test siderostats, and in October of the same year with the 8 meters Unit Telescopes (UTs). After more than one year of operation, it is now possible to evaluate its behavior and performances with a relatively long timescale. During this period, the technical downtime has been kept to a very low level. The most important parameters of the instrument (interferometric efficiency, mechanical stability,...) have been followed regularly, leading to a good understading of its performances and characteristics. In addition to a large number of laboratory measurements, more than 3000 on-sky observations have been recorded, giving a precise knowledge of the behavior of the system under various conditions. We report in this paper the main characteristics of the VINCI instrument hardware and software. The differences between observations with the siderostats and the UTs are also briefly discussed.
\end{abstract}

Keywords: infrared interferometry, VLTI, optical fibers, integrated optics, wavelets, commissioning.

\section{INTRODUCTION}

VINCI was the first interferometric instrument to be installed on Paranal. It is the result of a collaboration between the Observatoire de Paris (LESIA) for the design and construction, the Max-Planck Institut für Extraterrestrische Physik (Garching, Germany) for the infrared camera LISA, under the direction of the European Southern Observatory (ESO). As the first interferometric instrument to be installed at ESO, it has been a great source of experience.

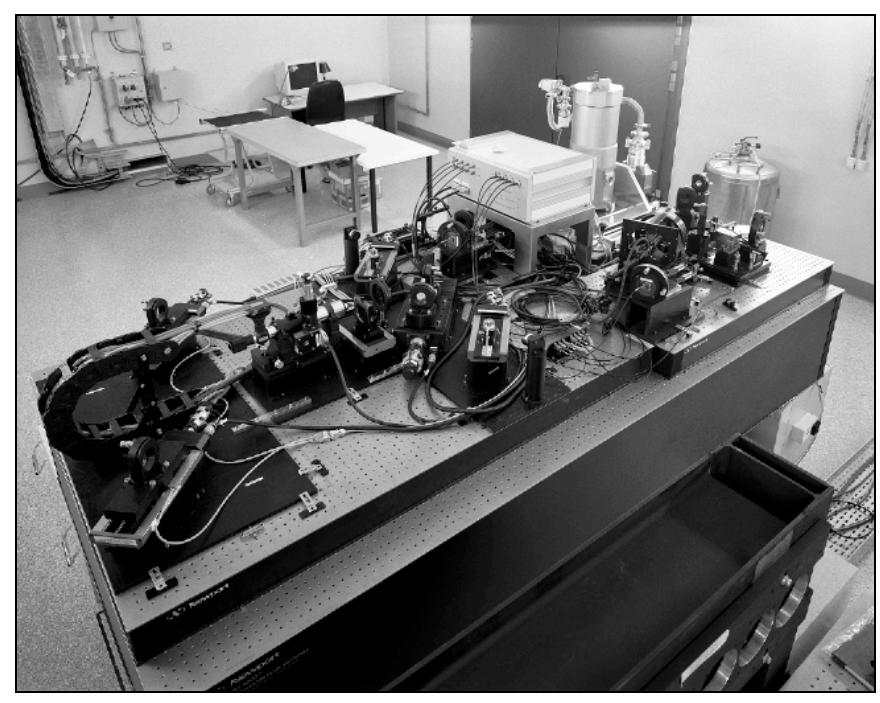

Figure 1. VINCI installed in the VLTI laboratory at Paranal.

*pkervell@eso.org, European Southern Observatory, Alonso de Cordova 3107, Casilla 19001, Vitacura, Santiago 19, Chile, tel. +5655435231 


\section{PRINCIPLE OF VINCI}

In its interferometric mode, VINCI operates mostly like $\mathrm{FLUOR}^{4}$, and produces interferograms modulated in optical path difference from stellar sources. The trajectory of the beams in the stellar interferometer mode is detailed in Figure 2. After the beams have been transported through the VLTI optical trains and delay lines down to the laboratory, they are folded onto the VINCI table, and then injected via fibers into an optical correlator, the MONA box. For a more detailed description of the hardware design of VINCI, the interested reader is referred to Kervella et al. ${ }^{5}$.

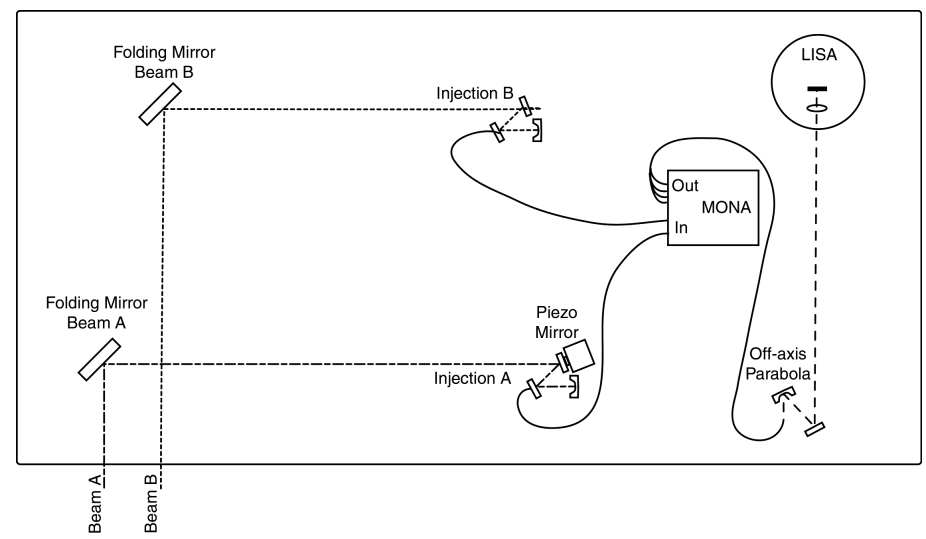

Figure 2. VINCI in stellar interferometer mode.

\section{HARDWARE PERFORMANCES IN THE LAB}

\subsection{Injection optics/mechanics}

VINCI relies on an original design of its injection optics to focus the stellar light on the injection fiber heads. It is based on an on-axis parabola, which is much less sensitive to small misalignments than the usual off-axis parabolas (FLUOR). The images produced by this optical setup show little aberration, and the injection efficiency achieved on VINCI is very consistent with the expected one. Using the motorized controls of the injection parabolas of VINCI, the positioning repeatability of the star image on the fiber head has been measured to be better than $0.3 \square \mathrm{m}$, for a fiber core diameter of $6.5 \square \mathrm{m}$. The resulting flux losses are therefore kept to a low level $(<5 \%)$.

\subsection{Triple coupler}

The beam combiner used on VINCI has been designed and built by the Le Verre Fluoré company (France). This device is based on single-mode fluoride glass fibers and couplers, and is operated in the photometric $\mathrm{K}$ band $(2.0-2.4 \square \mathrm{m})$. The waveguides are used to filter out the spatial modes of the atmospheric turbulence. The principle of the MONA box is shown on Figure 3. When used in Autotest mode, i.e. using an artificial light source located in the interferometric laboratory, it produces the type of interferogram presented on Figure 4.

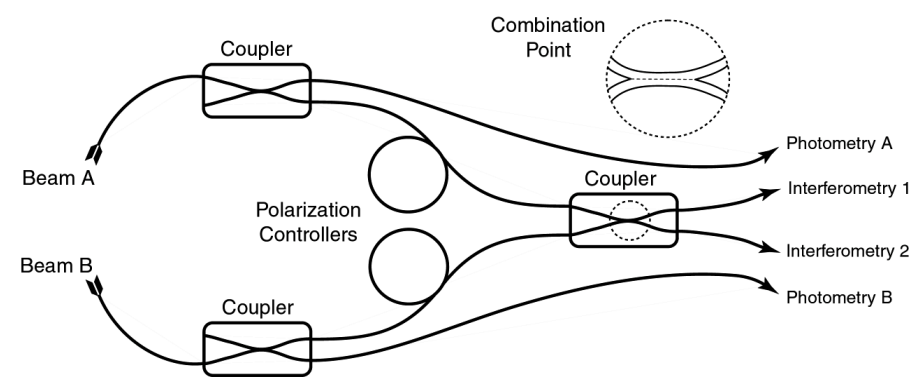

Figure 3. The fiber-based beam combiner of VINCI (MONA) 
The fluoride glass fibers used in the MONA beam combiner do not maintain the polarization of the incoming stellar light. Therefore, it is necessary to compensate for the polarization mismatch introduced by the input fibers inside the MONA box, before the combination point. This is achieved using two motorized polarization controllers (Figure 3) that can be individually adjusted. It is then possible to build a map of the instrumental contrast of the beam combiner in two dimensions (Figure 5), and to localize the maximum contrast in Autotest. The procedure is slower when optimizing the fringes contrast on sky, but the principle is the same.
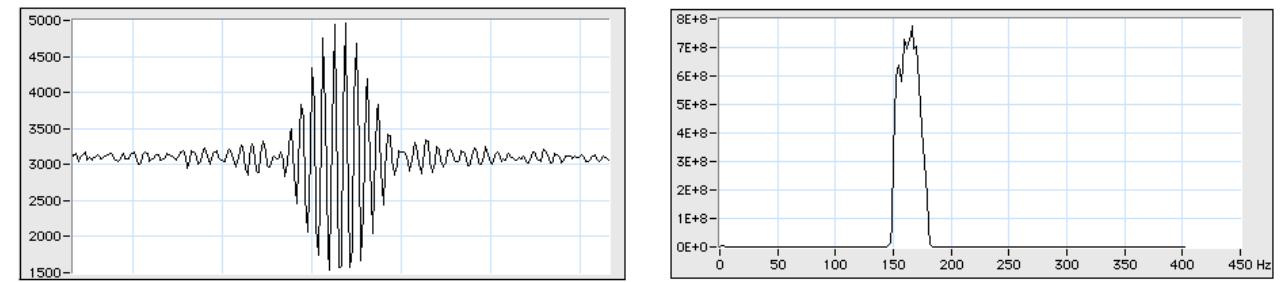

Figure 4. Fringe packet obtained in Autotest and the corresponding power spectral density.

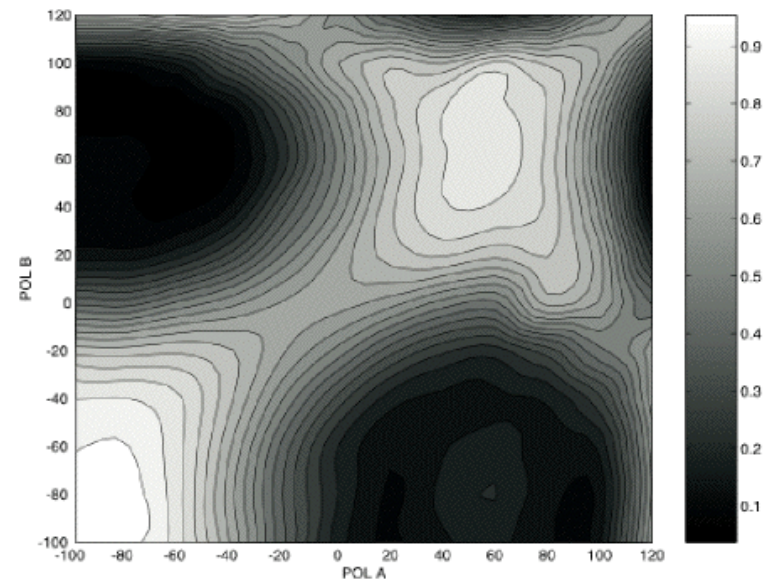

Figure 5. Map of the interferometric efficiency of MONA (in percentage of maximum) as a function of the position of the polarization controllers (arbitrary units).

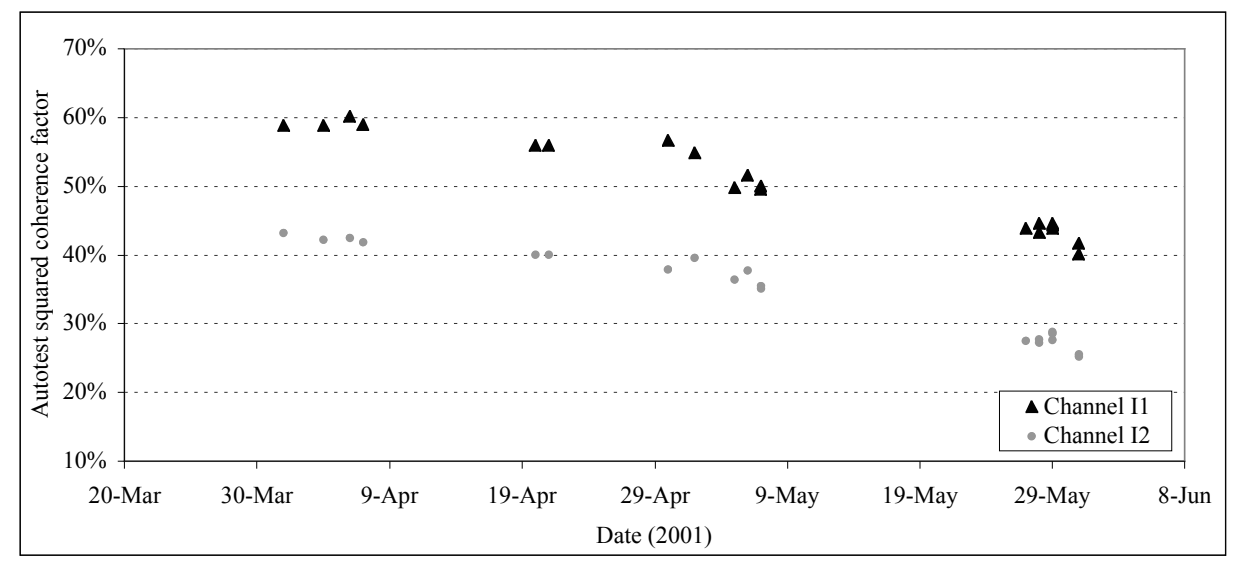

Figure 6. Decrease of the MONA interferometric efficiency in Autotest due to fiber cables contraction.

During the few months that followed first light with the siderostats (when interferometric efficiency was $87 \%$ on sky), a loss of interferometric efficiency of the MONA box was observed, both in Autotest and on sky. This loss was difficult to explain, but after some tests, it appeared that the polymer cables that are used to protect the fragile fluoride glass 
fibers were to blame. The thermal expansion coefficient of these cables is larger than the one of the fibers themselves. Therefore, when the temperature went down by a few degrees in the laboratory, the fibers suffered a mechanical stress that caused a severe birefringence (Figure 6). The separation of the two polarizations on the final interferograms (Figure 7) produced a very strong loss of contrast, and a strange double-peaked power spectral density (Figure 8) characteristic from the superimposition of the two polarizations fringe packets offset in OPD. This problem was solved by changing the path of the fiber cables, adding curves, therefore giving more space to the fibers inside the cable. After this repair, the contrast of the fringes obtained on sky went back to $70 \%$.

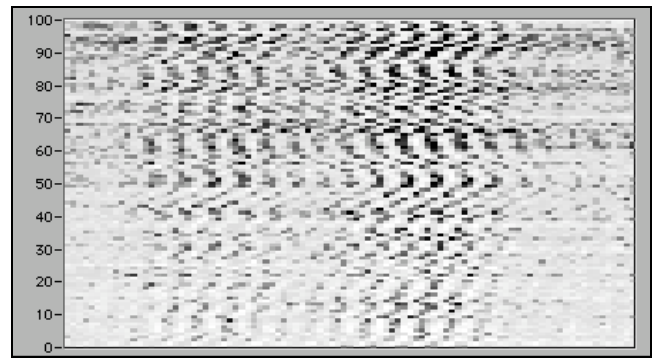

Figure 7. Series of interferograms obtained in Autocollimation mode showing the double peak feature characteristic of birefringence.

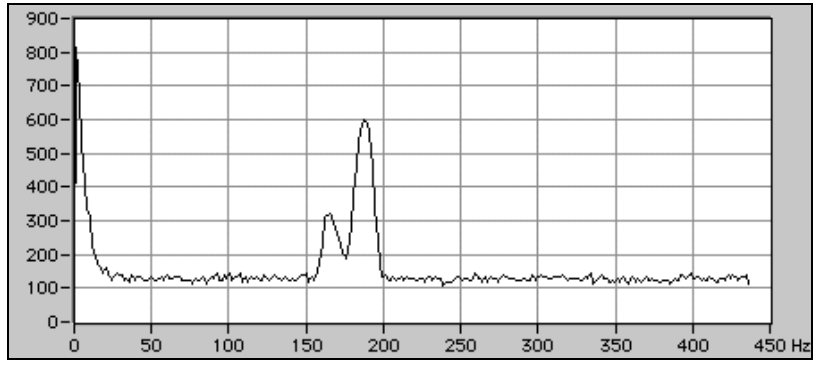

Figure 8. Average power spectral density of the interferograms presented on Figure 7 showing birefringence effect.

\subsection{Output stage}

The four fiber outputs of VINCI are imaged on four LISA camera photosites. The optics used for this purpose include an off-axis parabola to produce a collimated beam from the MONA fiber bundle, and an achromatic doublet located inside the camera dewar to focus the four images on the detector. The HAWAII detector used in LISA has the particularity to distributes part of the light falling on a single pixel over the four pixels located above, below, left and right of the target pixel. This process of diffusion causes a loss of $42 \%$ of the incoming signal from the target pixel ${ }^{7}$.

It has been shown experimentally that the percentage of incoming flux that is actually read out of a single pixel of LISA is more than $39 \%$ of the total light, for each of the four signals. This yields an optical fiber imaging efficiency of at least $67 \%$. The LISA camera has the possibility to read $2 \times 2$ or $3 \times 3$ pixels windows, but there is no gain in signal to noise ratio compared to a single pixel, as the gained flux is compensated by the additional readout noise.

\subsection{Overall Photometric transmission of VINCI}

When used in stellar interferometer mode, VINCI uses a very simple optical configuration (Figure 2), with only four mirror reflections for each arm before the injection in the optical fibers. The output of the four signals from MONA on the LISA camera has more optical elements (off-axis parabola, camera window,...). The main sources of flux loss are listed in Table 1. It is important to emphasize that the atmospheric turbulence is not considered here, and the average efficiency of the light injection is lower than the $78 \%$ stated here when observing on sky. The total photometric efficiency of VINCI (6.3\%) is defined here as the average number of photons recorded on the four windows of LISA compared to the total number of incoming photons from the two interferometer arms.

Table 1. Photometric transmission of VINCI.

\begin{tabular}{|l|l|}
\hline Optical element & Transmission \\
\hline Input mirrors (4) & 0.92 \\
\hline Fiber injection (ideal case) & 0.78 \\
\hline Transmission of MONA & 0.87 \\
\hline LISA window + cold doublet + K filter & 0.48 \\
\hline Energy in one LISA pixel & 0.39 \\
\hline Quantum efficiency LISA (K) & 0.62 \\
\hline TOTAL & $\mathbf{0 . 0 6 3}$ \\
\hline
\end{tabular}




\subsection{Commissioning tasks}

VINCI has performed a number of commissioning measurements on the VLTI infrastructure. The detailed description of the results of these tests is beyond the scope of this paper but for example, one can mention the verification of the performances of the delay lines using a K-band laser, and the measurement of the piston through relatively fast ( $>30$ $\mathrm{Hz}$ ) tracking of the fringes (Di Folco et al. ${ }^{6}$ ). Another role played by VINCI during the passed year has been to track the fringes on each new baseline commissioned, in order to constrain the optical path difference model of the VLTI. This was successfully achieved on four baselines up to now $(16 \mathrm{~m}, 46 \mathrm{~m}, 66 \mathrm{~m}$ and $103 \mathrm{~m}$ long), and will be pursued in the next years with the test siderostats to commission the numerous baselines of the Paranal platform. For further information on the VLTI commissioning, the interested reader is referred to Schöller et al. ${ }^{10}$.

\section{INFRARED CAMERA}

The infrared camera used in VINCI (called LISA) is built around an engineering-grade 1024x1024 pixels HAWAII array (Rockwell) presenting a large number of cosmetic and functional defects (dead quadrant, dead and hot pixels,...). As we are using only four pixels of the array, these defects fortunately do not affect our measurements.

\section{1. $\quad$ Readout noise}

Controlled by a standard IRACE controller (Meyer et al. ${ }^{3}$ ), the detector has shown a relatively low readout noise level, especially when using multiple A/D conversion. Figure 9 shows a map of the $64 \times 64$ pixels lower left part of the quadrant used in LISA, where grey scale coding corresponds to the readout noise level for single A/D conversion. The scale is linear from 0 to $25 \mathrm{e}^{-}$, the white positions corresponding to dead or hot pixels. The readout noises of the four pixels used in VINCI are $12 \mathrm{e}^{-}$(PA), $9 \mathrm{e}^{-}$(PB), $11 \mathrm{e}^{-}$(I1), $16 \mathrm{e}^{-}$(I2), in single-conversion mode. By using multiple A/D conversion of the pixel readouts, they are reduced by a factor of two, at the cost of a $20 \%$ lower maximum frequency.

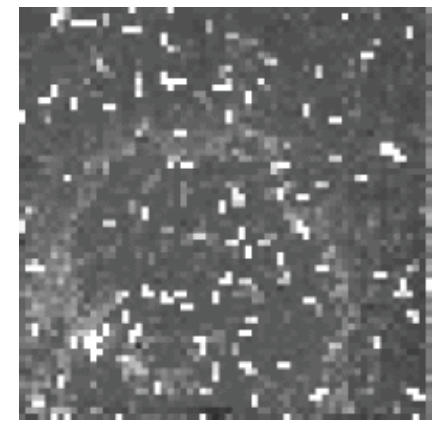

Figure 9. Noise map of LISA.

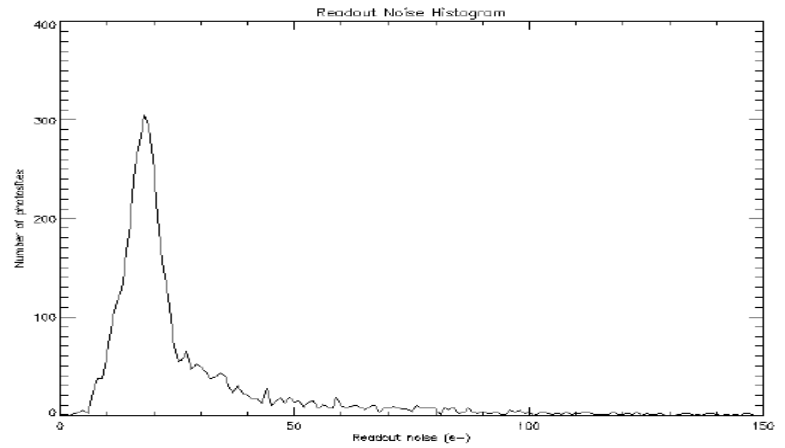

Figure 10. Histogram of LISA readout noises

\subsection{Modulation transfer function}

When recording a modulated signal, any memory effect on the detector can reduce the measured amplitude. This effect, harmful to the instrumental visibility of the fringes, was studied specifically in VINCI by checking the response of the detector to a chopped signal. Located very close to a single-mode fiber, the chopping wheel produced a shut-off time of $330 \square \mathrm{s}$, shorter than a single frame of the detector, read at a frequency of $2544 \mathrm{~Hz}$. The incoming signal can therefore be considered as a square modulated wave. The frequency response (modulation transfer function) of the camera is then deduced from the ratio of the output signal (Figure 11) to the input wave power spectra. The resulting modulation transfer function is presented on Figure 12 compared to a perfect integrator. The conclusion of these tests were that LISA camera behaves nearly like a perfect integrator (deviation of less than $10 \%$ at $1.2 \mathrm{kHz}$ ). 


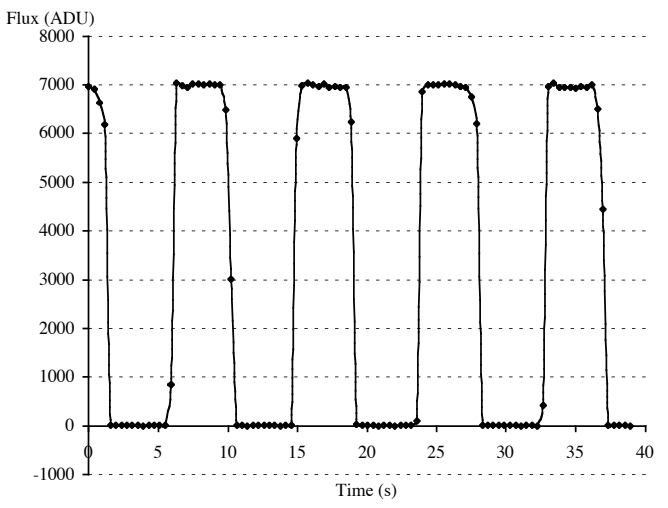

Figure 11. Chopped output signal of LISA.

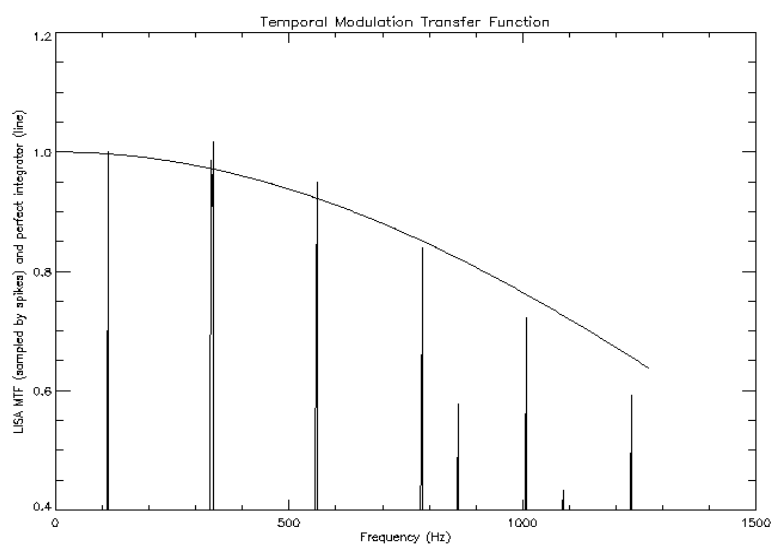

Figure 12. Modulation transfer function of LISA (peaks), compared to a perfect integrator (curve).

\section{SOFTWARE STRUCTURE}

\subsection{Observation preparation}

As with the other instruments of the VLT Observatory, the preparation of the observations is based on a several phases. Phase 1 concerns the initial proposal sent to the selection committee. Once the observation program has been accepted, then the astronomers are invited to prepare their observations in details during the Phase 2. This is done using a Javabased program called P2PP (Phase 2 Proposal Preparation tool). This program allows to define precisely the parameters of the observations (number of interferograms to be recorded, exposure time,...). The product of P2PP is a series of Observation Blocks (OBs) that are sent to Paranal, where they are executed by the Observing Software (OS).

The preparation of an interferometric observation is more complex than with a single-dish telescope. The changes in length and orientation of the projected baseline during the night (supersynthesis) create particular configurations that can either limit the observability of the object or produce interesting modulations of the actual spatial frequency of the observation. To prepare the observations, a prototype set of observation preparation tools (visibility calculator and exposure time calculator) is available on the ESO web site, at the following internet address:

http://www.eso.org/observing/etc/doc/indexdev.html.

\subsection{Fringes detection}

The first step for the recording of data is to detect the presence of the fringes in the OPD range scanned by the VINCI piezo mirror (up to $300 \square \mathrm{m}$ ). Two methods are used in VINCI to detect the fringes: the quicklook, and the peak integral.

The quicklook method is taken directly from the FLUOR instrument. This method allows both to detect and center the fringe packet in the scanned range. During the observations, a simple fringe packet centroid locator algorithm is applied to the data provided by LISA. The principle is to detect the pixels in the photometrically calibrated interferogram that have an intensity above a user-defined number of times the noise level (typically 7). The fringe packet center (in OPD) is measured with a precision of about one fringe $(2 \square \mathrm{m})$ after each scan and the resulting error is fed back to the VLTI delay lines as an OPD offset. This capability, called fringe coherencing, ensures that the residual OPD is less than a coherence length despite possible instrumental drifts. The correction rate (once per scan, i.e. up to about $50 \mathrm{~Hz}$ ) is too slow to remove efficiently the differential piston mode of the turbulence.

The peak integral is based on the incoherent accumulation of the fringe peak in the power spectral density of several interferograms. Each OPD position is scanned for a number of times (typically 10 to 100), and the detection occurs when the peak goes above a predefined level above the background noise level of the PSD. When the fringes are detected, it is not possible with this method to know their position inside the interferogram. This detection method is used to search for very faint fringes, that escape the classical detection using the quicklook. In practice, both detection 
methods are used in parallel, to benefit both from the centering capability of the quicklook and of the sensitivity of the peak integral.

A third fringe detection method is currently being investigated, based on the wavelets transformation. This should make it possible to obtain a better sensitivity to faint fringes, while keeping the coherencing capability (fringe packet centering).

\section{DATA PROCESSING}

\subsection{Fourier transform processing}

When the turbulent beams are spatially filtered by the single-mode waveguides, their random phase corrugations are transformed into intensity fluctuations which are monitored by the photometric signals coming out of MONA. It is then possible to correct each interferogram from these fluctuations and produce highly stable fringe visibility measurements that are independent of the spatial modes of the atmospheric turbulence.

Squared coherence factors $|\mu|^{2}$ (modulus of the complex coherence factor between the two beams) are computed from the normalized energy of the fringe signal in the power spectra of each corrected interferogram, and averaged over a series of a few hundred scans to reduce the statistical noise. The interested reader can find a detailed description of this Fourier transform-based processing algorithm in Coudé du Foresto et al. .

\subsection{Wavelets transform processing}

When dealing with the classical Fourier transform (FT) and especially with a quadratic estimator like $|\mu|^{2}$ (corresponding to the energy of the spectral density located at the fringe frequency) the debiasing step is critical and may completly spoil the compution of $|\mu|^{2}$. Among all biases, several are easely removed (white noise), like photon noise and detector noise. Other biases like the ones introduced by photometric fluctuations, differential piston or instrumental spikes are almost impossible to remove using the classical Fourier analysis, therefore requiring a timefrequency approach.

The most direct method for this purpose is the continuous wavelet transform (WT) using the Morlet wavelet (gaussian envelope times plane wave function). The main advantage of this wavelet is that it looks like the VINCI interferograms. It is thus extremely efficient to locate in both time and frequency domains the energy of the fringes. Such an approach, allows to compute the energy of the fringe packet without adding biases that are located outside of the fringe packet in OPD (optical path difference, also time domain) but that are located at the fringes frequency.

Figure 13 illustrates the advantage of the WT compared to the FT. The recorded photometry is very low a few microns from the beginning of the interferogram. The calibration of the interferogram by the photometry (division by $\sqrt{P_{A} \cdot P_{B}}$ ) strongly amplifies the noise in the low photometry region (Figure 13b). In the Fourier domain, the effect is spread over all frequencies and recovering the visibility becomes difficult. In wavelets analysis (the WT of the calibrated interferogram is presented on Figure 13c), the amplified noise is not taken into account since the energy is only integrated locally in the OPD and frequency domain as seen on Figure 13d (thick curve).

The wavelets analysis has also the remarkable ability to identify interferograms affected by atmospheric piston, therefore allowing to remove them during the data processing (Segransan ${ }^{9}$ ). Thanks to the efficient rejection of the noise in the WT, it is also possible to detect fringes in very low signal to noise conditions. It is foreseen in the near future to implement a dedicated WT based algorithm for the real-time detection of the fringes during the observations. A WT module has been added to the VINCI data reduction software based on the work from Segransan ${ }^{8,9}$ in addition to the classical FT algorithm. 


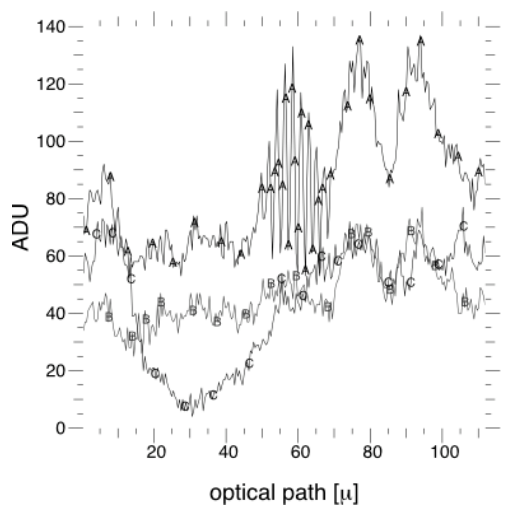

(a)

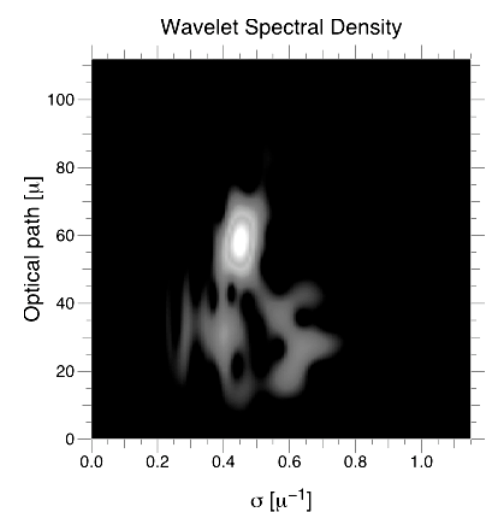

(c)

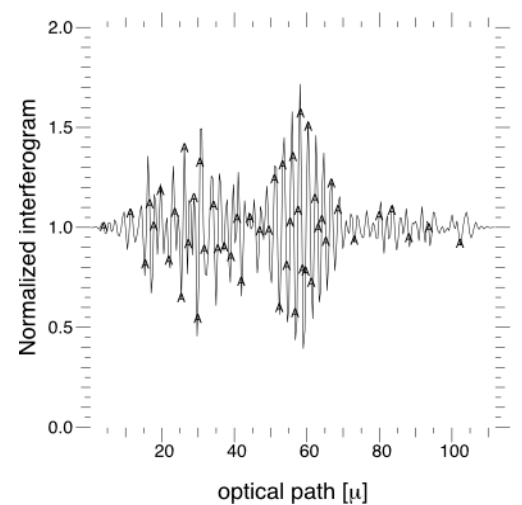

(b)

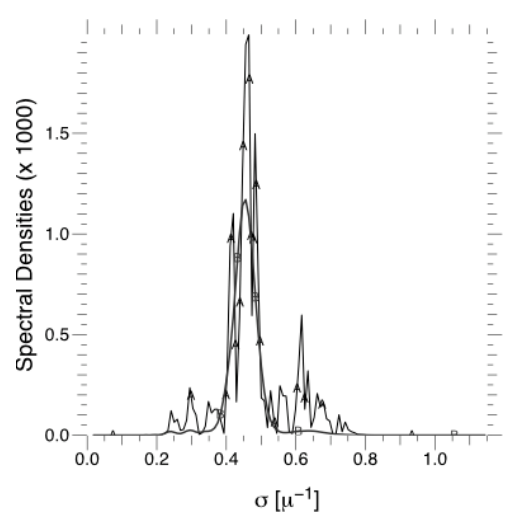

(d)

Figure 13. Compared integration of the fringe power between Fourier and Wavelets methods, on VINCI data obtained with UT1-UT3. Figure 13a shows the raw signals I1, PA and PB. Figure 13b is the calibrated interferogram. On Figure

$13 \mathrm{~d}$, the Fourier power spectral density is the thin line, and the wavelets spectrum (Figure 13c) projected along the frequency axis is the thicker, gaussian shaped curve.

\subsection{Calibration and Data analysis}

The analysis of the processed fringes data is done using a toolbox programmed in IDL. This interface allows the user to select the interferograms based on a combination of criteria, such as the photometric signal to noise ratio or the strength of the piston effect. From the processed data files, this software outputs calibrated visibility values that can then be compared to an astrophysical model of the observed target or directly used to derive its angular size.

\section{ON-SKY OBSERVATIONS}

\subsection{Siderostats}

On-sky interferometric observations with the siderostats have been going on continuously at Paranal since the first fringes obtained on March 19, 2001. The first series of interferograms recorded with the VLTI at this occasion is presented on Figure 14 (time is from bottom to top, one line per interferogram). The fringes were tracked and centered by VINCI, therefore removing part of the atmospheric piston. 


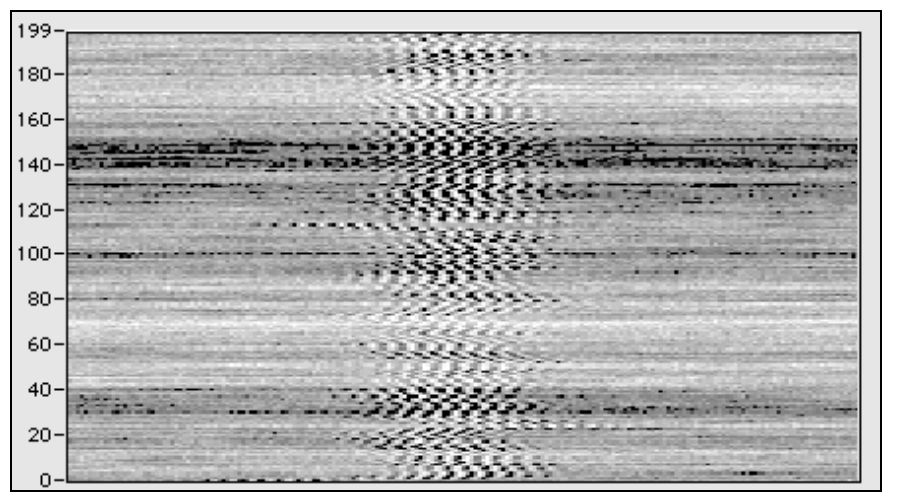

Figure 14. First interference fringes of the VLTI on $\square$ Hya, X axis is OPD (one fringe equals $2.2 \square \mathrm{m}$ ), Y axis is the scan number in the series (about 90 seconds of data in total)

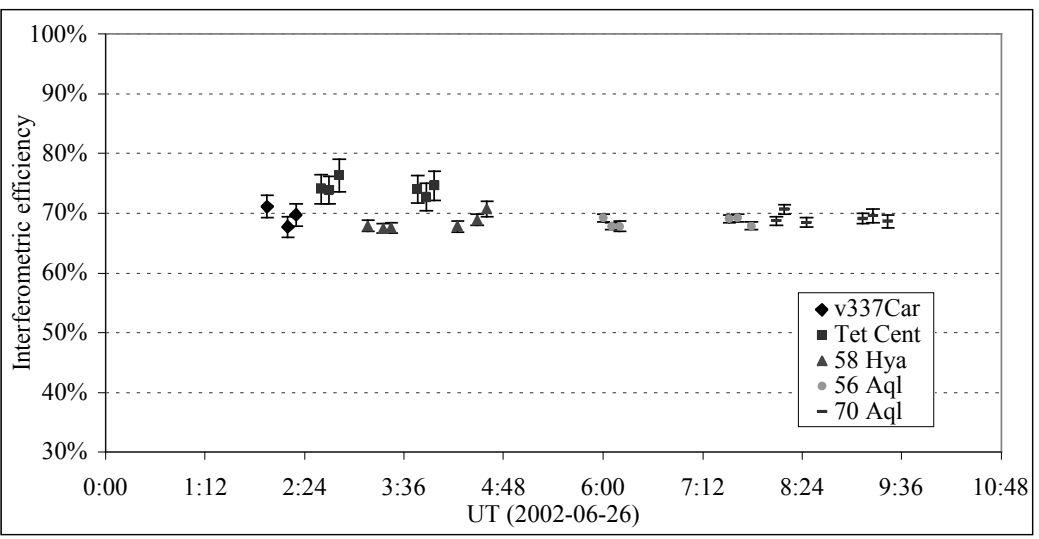

Figure 15. Interferometric efficiency of VINCI and the VLTI test siderostats on sky (66 m baseline, June, 2001)

Over a long timescale, the interferometric efficiency of the MONA beam combiner has evolved substantially, due to the sensitivity of the MONA couplers to temperature (see the discussion in Sec. 3.2). Nethertheless, on a timescale of one night, the interferometric efficiency measured on-sky has been stable, as shown on Figure 15. The excess efficiency visible on the two series of transfer functions computed from the $\square$ Cen data comes apparently from an overestimation of the a priori angular size of this star taken from a calibrators catalog. The average interferometric efficiency over this night was $69 \pm 2 \%$ ( $\square$ Cen data excluded).

\subsection{Unit telescopes}

After the first fringes with the VLTI test siderostats obtained in March 2001, the first recombination of the light from two 8-meter telescopes of Paranal was achieved in October of the same year. Interference fringes between the star light collected by the telescopes Antu (UT1) and Melipal (UT3), separated by 102.5 meters, were recorded by VINCI. One major surprise of these first observations was that the flux injected in the optical fibers of VINCI was much more stable that initially expected (Figure 16). The common view of the injection of a multi-speckle star image in an optical fiber was initially that a single moving speckle is injected at a time, and that the flux can be completely lost between speckles. In reality, it was observed that light is present on LISA more than $95 \%$ of the time.

The interferometric transfer function of the VLTI/VINCI during the first UT1-UT3 commissioning run was measured to be $61 \%$. It should be stressed here that the positions of the polarization controllers of MONA were not optimized, and this value is therefore not optimal and not comparable to the transfer function obtained on the siderostats. 


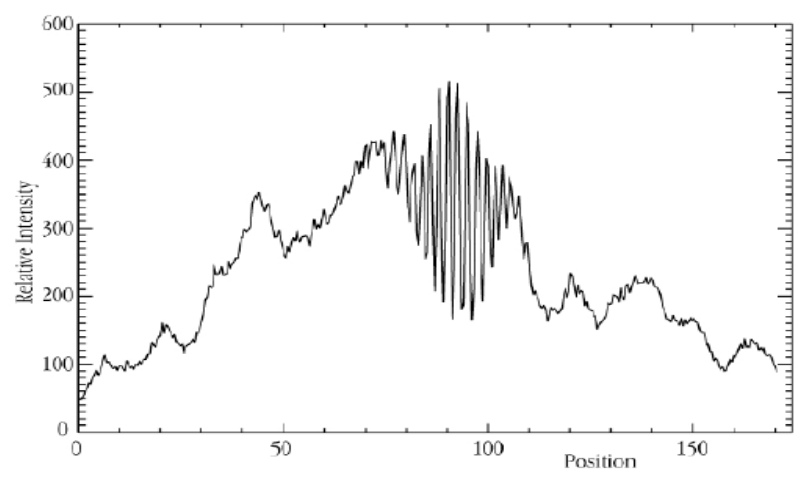

Figure 16. Raw interferogram of $\square$ Geminorum obtained with UT1-UT3

A number of stars were observed during the four runs of commissioning with the UTs. The faintest object on which fringes were acquired was the main sequence star HD 112282. Observed under Paranal median seeing conditions $(0.6$ to 0.7 " at $\square=0.5 \square \mathrm{m}$ ), it was possible to track the fringes efficiently on this $\mathrm{K}=7.7$ unresolved star, with a signal to noise ratio better than 10 on the interferometric channels, yielding a limiting magnitude better than $\mathrm{K}=9$. A relatively precise value of the transfer function of the interferometer could even be derived from this observation.

\subsection{Precision vs. magnitude}

The visibility estimators used for the processing of the data of VINCI are quadratic (Coude du Foresto et al. ${ }^{1}$ and Segransan ${ }^{9}$ ), and therefore the final precision of the measurement is expected to be proportional to the correlated magnitude CorrK of the object, defined as the following:

$$
\operatorname{Corr} K=\square 2.5 \log _{10} 0^{\square} 0^{0^{\frac{m a g}{2.5}}} \cdot \square^{2} \stackrel{\square}{=}
$$

where $\operatorname{magK}$ is the photometric magnitude of the star and $\square^{2}$ is the squared coherence factor. Using the observations obtained on sky with the siderostats, it was possible to quantify the precision achievable with VINCI as a function of the correlated magnitude (Figure 17).

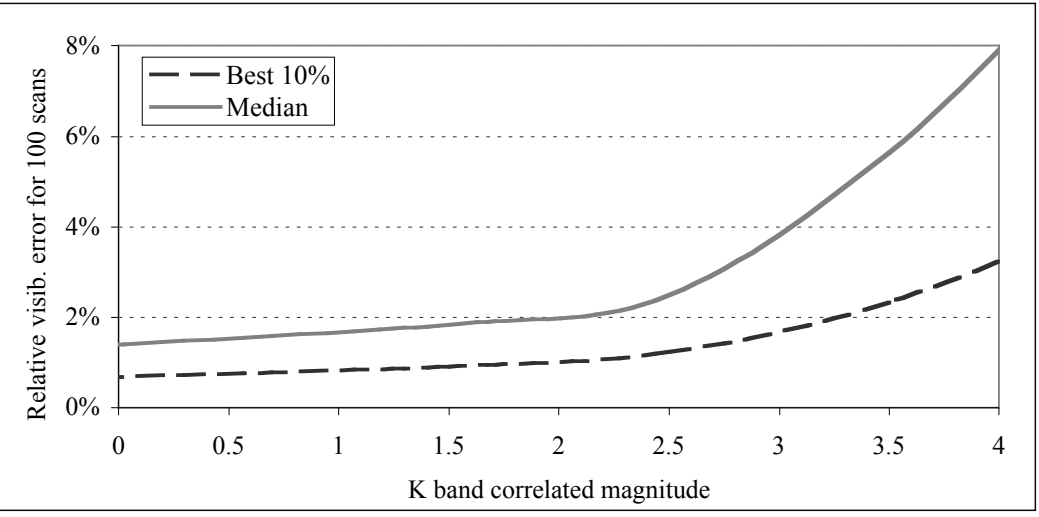

Figure 17. Accuracy of VINCI visibility measurements with the VLTI test siderostats.

The precision does not increase for the brightest sources because of the atmospheric piston effect, not yet corrected on the VLTI by a fringe tracker. Once the FINITO fringe tracker will be installed, the removal of the fringe motion will allow to reach a much better precision. Laboratory measurements at Paranal without piston and in photon shot noise limited regime produced relative precisions (FT estimator) better than $0.05 \%$ on the visibility values for 100 interferograms. This value should be the limit of precision achievable with the FT based quadratic visibility estimators. 


\subsection{Observed stars}

During the commissioning of the VLTI, a number of objects have been observed, with the siderostats or with the 8meter telescopes UT1 and UT3. The list of all observed targets is presented in Table 2. Most of the stars listed have been observed several times, and the number of fringe files is indicated after the name of the object. See also:

http://www.eso.org/projects/vlti/instru/vinci/vinci_data_sets.html

Table 2. Stars observed with VINCI until February 2002.

\begin{tabular}{|c|c|c|c|c|c|c|}
\hline Star ( $\#$ Observ.) & alf Sco (19) & del Crv (2) & H Sco (3) & nu Hya (48) & S Ori (23) & V Hya (3) \\
\hline $1 \mathrm{Oph}(112)$ & alf $\operatorname{Ser}(5)$ & del Eri (9) & HD $100453(2)$ & nu Pav (8) & $\mathrm{S} \mathrm{Sel} \mathrm{(23)}$ & v2111 Oph (6) \\
\hline 1 Pup (5) & alf Tau (4) & del Sgr (9) & HD 10550 (8) & NZ Gem (3) & sig Lib (109) & V3872 Sgr (5) \\
\hline $2 \operatorname{Sex}(3)$ & AR Cet (13) & del02 Gru (4) & HD 112282 (2) & omi Cet (45) & sig Pup (61) & v744 Cen (2) \\
\hline $24 \mathrm{PsA}(7)$ & BD-04 782 (3) & eps $\mathrm{Crv}(56)$ & HD 20234 (9) & phi1 Lup (3) & SW Vir (13) & V806 Cen (33) \\
\hline $25 \mathrm{CMa}(27)$ & bet $\operatorname{Car}(3)$ & eps Eri (14) & HD 216149 (11) & phi2 Ori (4) & SWR 69 (8) & v919 Cen (2) \\
\hline 39 En (22) & bet Cen (13) & eps Lep (137) & HD 787 (6) & pi Eri (15) & T Ari (19) & VY Leo (9) \\
\hline 43 Vir (48) & bet Cet (293) & eps Peg (127) & HD 83443 (4) & pi Hya (8) & $\mathrm{T} \operatorname{Cet}(30)$ & VZ Pic (9) \\
\hline 6 Pup (10) & bet $\mathrm{Cne}(15)$ & eps $\operatorname{Ret}(8)$ & HR 2447 (3) & pi Leo (2) & T Eri (5) & W Cen (7) \\
\hline AK Hya (4) & bet $\operatorname{Cor}(6)$ & eps Sco (177) & HR 4354 (2) & psi Phe (44) & T Lep (39) & W Hya (14) \\
\hline alf Ant (4) & bet $\operatorname{Crv}$ (3) & eta Ara (5) & HR 5152 (3) & $\mathrm{Q} \operatorname{Car}(2)$ & tau Aqgr (6) & W Ori (7) \\
\hline alf Aq̣l (5) & bet Dor (29) & eta Car (36) & HR $6682(4)$ & R Aqg (17) & tau Pup (3) & $\mathrm{X}$ Cne (3) \\
\hline alf Cen A (38) & bet Gru (254) & eta Cet (8) & kap Lib (4) & R Cne (29) & tau Pup (3) & X Hya (3) \\
\hline alf Cen B (6) & bet Ori (13) & eta Gem (6) & KP Pup (2) & $\mathrm{R} \mathrm{Crt}(3)$ & tau04 Eri (2) & $Y$ Cen (2) \\
\hline alf Cen C (2) & bet $P i c(10)$ & eta $\mathrm{Sgr}(147)$ & KQ Pup (8) & R Hya (3) & tet Cen (227) & YY Psc (6) \\
\hline alf Cet (42) & $C^{*} 284(13)$ & FU Ori (12) & $1 \operatorname{car}(9)$ & R Leo (45) & tet $\mathrm{Cma}(8)$ & zet Gem (20) \\
\hline alf $\mathrm{CMa}(625)$ & CE Tau (14) & gam Aq̣l (60) & LO2 Pup (28) & R Lep (34) & TW Hor (10) & zet Hya (5) \\
\hline alf $\mathrm{CMi}(8)$ & chi Aq̣r (13) & gam Cru A (20) & lam Aqqr (44) & $\mathrm{RPeg}$ (5) & TX Psc (4) & \\
\hline alf Eri (43) & chi Phe (14) & gam Eri (231) & lam Vel (25) & R Scl (17) & U Ant (4) & \\
\hline alf $\mathrm{Her}(8)$ & CI On (10) & gam Sco (4) & mu Gem (3) & R Vel (8) & U Ori (15) & \\
\hline alf Hya (271) & $\operatorname{del~CMa~(35)~}$ & gam Sge (12) & mu Hya (7) & RX Lep (48) & ups $\operatorname{Cet}(2)$ & \\
\hline alf Phe (3) & del Crt (8) & gam02 Vol (22) & NSV 14420 (7) & $S$ Lep (6) & ups $\operatorname{Lib}(6)$ & \\
\hline
\end{tabular}

\section{INTEGRATED OPTICS}

During a seven nights run on the VLTI (July 17-23, 2002) done in collaboration between ESO and LAOG (Observatoire de Grenoble, France), we tested on VINCI an integrated optics two-telescopes beam combiner, IONIC.
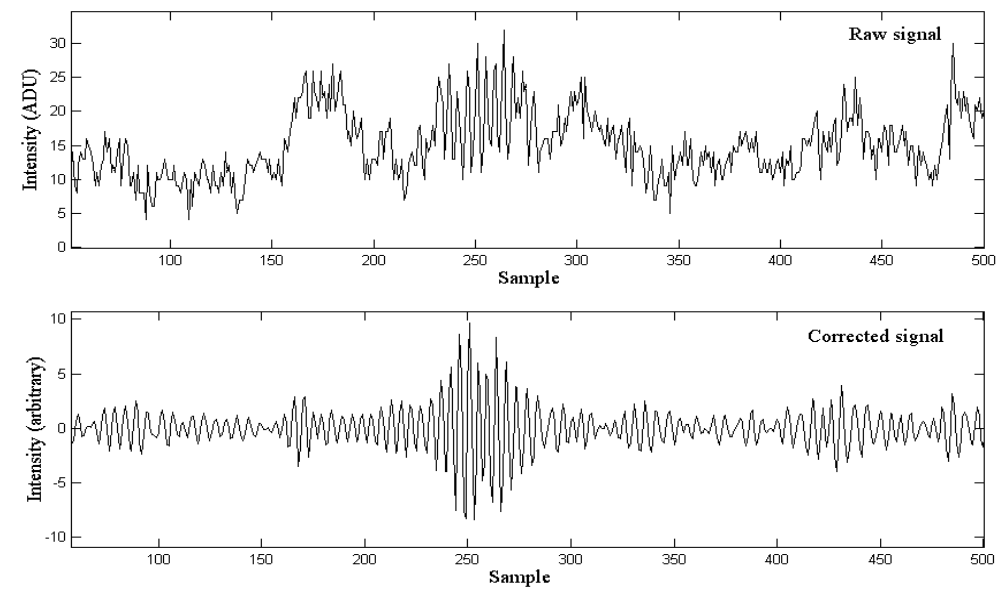

Figure 18. Fringes obtained on Altair $(\mathrm{H}=0.25)$ with the IONIC beam combiner installed on VINCI

This type of component had already been tested on sky at the IOTA interferometer in November 2000 (Berger et al. ${ }^{1}$ ). For this first run at Paranal, the installation of IONIC was realized without any modification of the optical setup of VINCI and only minor software changes were required (due to the single interferometric output of the component while the usual beam combiner MONA has two such outputs). The faintest star observed so far with the siderostats is HD 720 with an $\mathrm{H}$ magnitude of 3.1. It is currently difficult to determine the maximum reachable magnitude due to the 
mismatch in numerical aperture between the $\mathrm{H}$ band optical fibers of IONIC and VINCI feeding optics (optimized for fluoride glass fibers). To obtain the maximum transmission, it would require to change the injection optics of VINCI to adapt them to the numerical aperture of the IONIC fibers ( 0.12 compared to 0.23 for MONA fibers).

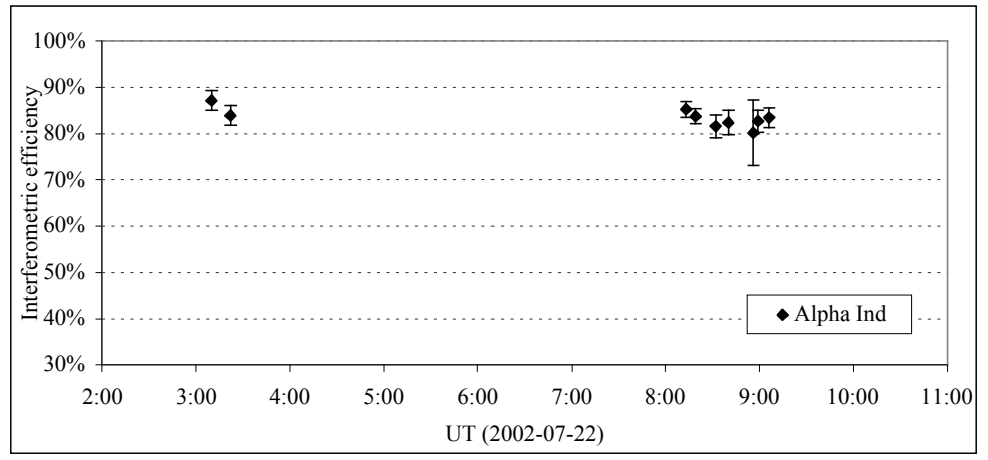

Figure 19. Interferometric efficiency measured on sky with the IONIC component installed on the VLTI.

Fringes were obtained on sky in the $\mathrm{H}$ band (1.5-1.8 $\square \mathrm{m})$, and their visibility was estimated with a relative accuracy of about $1 \%$. The IONIC component interferometric efficiency previously measured at LAOG is $90 \%$ for unpolarized light. As shown on Figure 19, the interferometric efficiency of VLTI+IONIC measured on sky was better than $80 \%$ on the star $\square$ Ind (uniform disk angular diameter of $3.28 \pm 0.04$ mas, effective wavelength of $1.58 \square \mathrm{m}$ ).

\section{CONCLUSION}

After the first fringes obtained with the VLTI test siderostats and the 8 meters Unit Telescopes, VINCI has demonstrated a high productivity, both in terms of commissioning results and scientific observations of stars. It has recently been opened to proposals from the European astronomical community. While new algorithms are being developed to exploit fully the potential in precision of this instrument (wavelet transform,...), the promising results obtained with the IONIC beam combiner open up exciting new perspectives of multiple telescopes beam combination (Kern et al. ${ }^{11}$ ), using the technology and experience gained from VINCI.

\section{REFERENCES}

1. V. Coudé du Foresto, S. Ridgway and J.-M. Mariotti, "Deriving object visibilities from interferograms obtained with a fiber stellar interferometer”, A\&A Suppl. Ser. 121, pp. 379-392, 1997

2. J.-P. Berger, P. Haguenauer, P. Kern, K. Perraut, F. Malbet, I. Schanen, M. Severi, R. Millan-Gabet, W. Traub, "Integrated optics for astronomical interferometers, IV. First measurements of stars", A\&A 376, p. 31, 2001

3. M. Meyer, G. Finger, H. Mehrgan, G. Nicolini, J. Stegmeier, "ESO infrared detector high-speed array control and processing electronic IRACE”, SPIE 3354, p. 134, 1998

4. V. Coudé du Foresto, G. Perrin, C. Ruilier, B. Mennesson, W. A. Traub, M. G. Lacasse, “FLUOR fibered instrument at the IOTA interferometer”, SPIE 3350, p. 856, 1998

5. P. Kervella, V. Coudé du Foresto, A. Glindemann, R. Hofmann, "VINCI: The VLT Interferometer Commissioning Instrument", SPIE 4006, p. 31 , 2000

6. E. Di Folco, B. Koehler, P. Kervella, V. Coudé du Foresto, et al., “Atmospheric and internal turbulence measured on the Very Large Telescope Interferometer with VINCI”, These Proceedings, SPIE 4838-35, 2002

7. G. Finger, European Southern Observatory, private communication, 2001

8. D. Segransan, Ph.D. Thesis, Université de Grenoble, 2001

9. D. Segransan, in preparation, 2002

10. M. Schöller, P. Gitton, et al., "Commissioning of the VLT Interferometer: from first fringes towards a general user facility”, These Proceedings, SPIE 4838-153, 2002

11. P. Kern, F. Malbet, J.-Ph. Berger, P. Haguenauer, K. Rousselet-Perraut, C. Perrier-Bellet, "Increasing the imaging capabilities of the VLTI using integrated optics”, These Proceedings, SPIE 4838-76, 2002 